\title{
A Strategy for the Rapid Discovery of Disease Markers Using the MassARRAY ${ }^{\mathrm{TM}}$ System
}

\author{
Charles P. Rodi ${ }^{1}$, Brigitte Darnhofer-Patel ${ }^{2}$, Patrick Stanssens ${ }^{3}$, Marc Zabeau ${ }^{3}$, and Dirk van den Boom ${ }^{2}$ \\ ${ }^{1}$ Rodi Pharma, San Diego, CA, USA; ${ }^{2}$ SEQUENOM, San Diego, CA, USA; and ${ }^{3}$ Methexis Genomics, Ghent, \\ Belgium
}

BioTechniques 32: S62-S69 (June 2002)

\section{INTRODUCTION}

Using information from the Human Genome Project and strategic relationships, SEQUENOM has established a portfolio of nearly 400000 assays for single nucleotide polymorphisms (SNPs) from the company's database of over 1.9 million designed SNP assays (available through the RealSNP.com ${ }^{\mathrm{TM}} \mathrm{Web}$ site). Because of their abundance, stability, and simplicity (almost all SNPs are biallelic), SNPs are extremely useful and valuable for conducting large-scale studies of human diversity, such as association studies, linkage studies, and pharmacogenomics $(2,4,6,7)$. Coupled with the high-throughput and accuracy of the MassARRAYTM System, such studies are possible at an unprecedented scale, speed, and economy.

The strategy described here is one of large-scale association studies. From the RealSNP.com Web site, coding sequence SNPs (cSNPs) are chosen. Using pools of DNAs made from the populations one wishes to compare, relative allelic frequencies are determined for the collection of cSNPs using the homogeneous MassEXTEND ${ }^{\mathrm{TM}}$ (hME) assay coupled with real-time analysis of the area under the mass peak of each allele (1). This allows one to pre-screen tens of thousands of SNPs at a reasonable cost, taking forward into individual genotyping only those SNPs that show a significant difference between the pools.

The SNPs selected in the pre-screen may merely be associated with the phenotype of interest rather than causing it. To increase the probability of finding the $\mathrm{SNP}(\mathrm{s})$ that cause a particular disease - should they exist - an effort is made to identify all the SNPs in the regions of interest and include them in the individual genotyping. Known SNPs in the regions are identified by once again searching the RealSNP.com Web site. Unknown SNPs are discovered and localized using base-specific fragmentation, a new application for the MassARRAY System developed by SEQUENOM in collaboration with Methexis Genomics (Ghent, Belgium). This collection of SNPs is then designed into multiplexed sets of hME reactions using SpectroDESIGNER ${ }^{\mathrm{TM}}$ software and is used for high-throughput genotyping of the individual DNAs from the two populations of interest.

This strategy allows for large numbers of SNPs to be evaluated as markers for disease genes, unknown SNPs to be discovered, and a precise determination of genotypes for each individual in the two phenotypically-defined populations, all using a single platform.

\section{BASIC TECHNOLOGY}

The MassARRAY system is a MALDI-TOF MS (matrix-assisted laser desorption/ionization time-of-flight mass spectrometry)-based system optimized for the automated analysis of nucleic acids (3). The basic aspects of the technology are shown in Figure 1, which depicts a typical homogeneous MassEXTEND assay, which is run in a 384-well microtiter plate (10). In a single-tube reaction, the DNA containing the SNP is amplified; then a sequence-specific hME primer is annealed immediately adjacent to the polymorphic site. The hME primer is extended in the presence of three dideoxyribonucleotide triphosphates (e.g., ddATP, ddCTP, and ddTTP) and one deoxyribonucleotide. The selection of the deoxyribonucleotide is made such that it will correspond to one of the alleles (e.g., dGTP). Three mass peaks are possible in the spectrum: unextended primer $(\mathrm{P})$; a single nucleotide extension specific for one allele (A1); and a primer extended by two or more nucleotides specific for the other allele (A2). The minimum mass difference seen between any two products will be at least the mass of a single nucleotide, a difference in mass that is approximately 100 -fold greater than needed to differentiate among the peaks. Following the extension reaction, SpectroCLEAN ${ }^{\mathrm{TM}}$ is added to the same tube to condition the sample prior to dispensing to a SpectroCHIP ${ }^{\mathrm{TM}}$, the launching pad for analyte into the mass spectrometer.

SpectroCHIPs are highly precise and uniform silicon chips pre-spotted with matrix that enable the routine MALDI-TOF MS analysis of nucleic acids (5). Two formats are currently available, a 96-element chip and a 384-element chip, which matches the format of a typical hME reaction plate. A minute volume (approximately 10 nanoliters) from each reaction is automatically transferred to a corresponding matrix pad. Up to ten SpectroCHIPs, each containing 96 or 384 reactions, may be inserted in the mass spectrometer at one time.

Once inside the MALDI-TOF MS, each matrix pad is irradiated by multiple laser bursts, volatilizing the matrix, and suspending the DNA analytes. Momentum is induced via a brief electric field, and the DNA molecules fly down a one-meter long path to a detector. The time of flight to the detector is proportional to the mass of the molecule. Thus, small masses make the journey quickly, while larger masses take longer. The timing of the flight is so precise that analytical accuracy is about $0.1 \%$ to $0.01 \%$. Fast, automated calling of alleles is done in real time, allowing for geno- 
typing results to be recorded about once every three seconds. Thus in under 4 hours of walk-away performance, 3840 SpectroCHIP elements can be interrogated. The number of genotypes possible will depend on the level of multiplexing, typically 1-plexes to 6plexes or 3840 to 23040 genotypes, respectively.

\section{DETERMINATION OF ALLELIC FREQUENCIES IN POOLED DNA SAMPLES}

The assay and analysis just described is an extremely accurate, fast, and affordable method for determining genotypes, but it can also be used to determine allelic frequencies within pools of individual genomic DNAs. This requires precise determination of DNA concentration for each individual and precise transfer of equal amounts of DNA in order to make representative pools. Typically, 90 to 300 individuals are included in each pool for which an allelic frequency is to be determined.

Figure 2 displays estimates of allelic frequencies determined by using pooled DNA versus precise allelic frequencies determined by genotyping each individual used to make the pool. As can be seen for both uniplex assays (Figure 2A) and multiplex assays (Figure 2C), the deviation from linearity indicates that the initial correspondence is not particularly good. This is believed to be the result of differential amplification of the alleles. A correction factor can be determined for each of the 192 assays examined simply by running each assay on an appropriate heterozygote. This establishes the ratio of skewing for each allele and allelic frequencies for the pooled samples can be adjusted. Corrected allelic frequencies from pools versus the allelic frequencies determined by genotyping are displayed in Figures $2 \mathrm{~B}$ and $2 \mathrm{D}$ for uniplex and multiplex reactions, respectively. $\mathrm{Al}-$ though application of correction factors greatly improves the data, in practice, it is not necessary to do so. This is because the differential amplification of alleles is systematic and consistent for any particular assay. Thus, when two pools are compared, the skewing of allelic frequencies does not affect the deltas (if any) observed between pools. Deltas can be determined with confidence and specific assays selected for the step of genotyping each individual represented in each pool.

\section{SNP DISCOVERY BY BASE-SPECIFIC FRAGMENTA- TION OF AMPLIFIED DNA}

Base-specific fragmentation represents a paradigm shift in the resequencing of nucleic acids. Resequencing used in this context means the comparison of an experimentally determined sequence with a reference sequence. With reference sequences now available for the human genome, as well as a growing collection of sequences from other species, resequencing is an ideal method for discovering genetic variation, validating sequences,

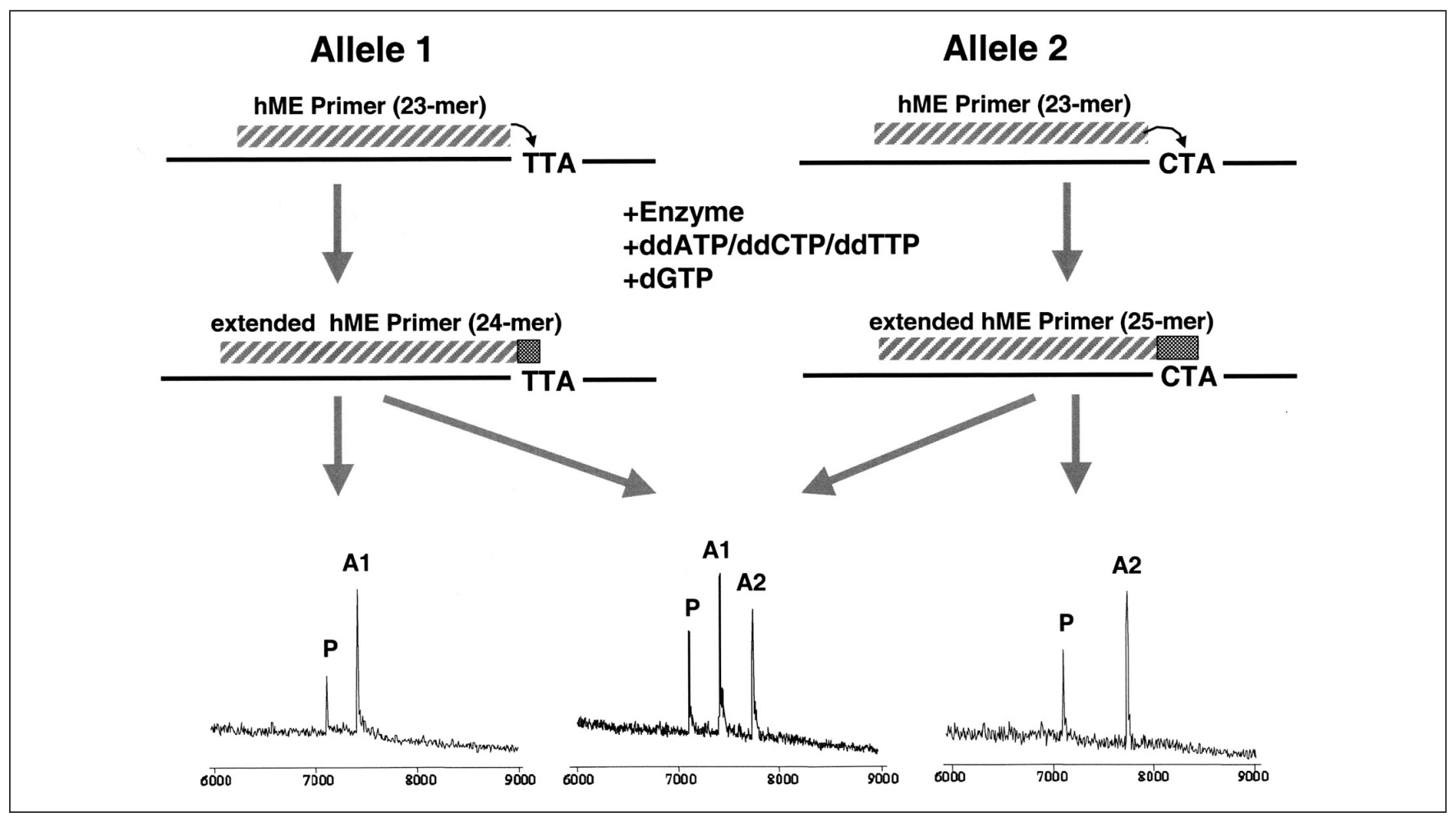

Figure 1. The homogeneous MassEXTEND reaction yields allele-specific products with approximately 100-fold more separation than is needed to distinguish them by MALDI-TOF MS. In this example of a C/T SNP, there are three dideoxyribonucleotides (ddA, ddC, and ddT) plus one deoxyribonucleotide (dG). The deoxyribonucleotide is the complement to one of the alleles in the amplified template. In this way, the extension of the primer annealed to the T-containing template is terminated immediately, resulting in a single-nucleotide addition. An identical primer annealed to the C-containing template is extended by two nucleotides since the first nucleotide inserted is the $\mathrm{dG}$, which allows the extension reaction to continue until ddA, $\mathrm{ddC}$, or ddT is inserted. The difference in mass between the two allelespecific extension is approximately $300 \mathrm{Da}$ in a portion of the mass spectrum where differences of $2 \mathrm{Da}$ to $3 \mathrm{Da}$ are resolvable. 
and performing diagnostics $(9,11)$. In the context of this article, it is ideal for the discovery and localization of unknown SNPs (among other genetic variants).

In conventional dideoxy sequencing, a sequence-specific primer is extended with a DNA polymerase in the presence of a mixture of dideoxyribonucleotides and deoxyribonucleotides. This creates nested sets of truncated fragments with a common $5^{\prime}$ end. Separation on a gel allows one to deduce the sequence from the sets of truncated sequences (8). In base-specific fragmentation, a single-stranded copy of the target sequence is created and in four separate reactions fragmented to completion at positions corresponding to each of the four bases. This reduces the nucleic acid to a collection of sets of oligonucleotides, which are easily resolvable with the precision, accuracy, and resolution of the MALDI-TOF MS. Using a reference sequence allows one to definitively identify each resulting peak. Changes in sequence have profound and easily discernible effects on the pattern of peaks produced. This is illustrated in Figure 3. In this example an $\mathrm{A} / \mathrm{C}$ transversion is shown. Suppose the known (reference) sequence were the A-containing sequence; then one would expect that an A-specific cleavage of the displayed sequence would produce the two fragments shown, a 7-mer and a 6-mer (ignoring the end fragments). Now consider the result if a sample contained a $\mathrm{C}$ at the second A position. There would be only two A residues, and the cuts would produce the single large fragment shown, a 13-mer; the 7-mer and 6-mer would disappear (or in the case of a heterozygote, be diminished in intensity). The Cspecific cleavage would, of course, produce the converse result of a 13-mer for the A allele and a 6-mer plus a 7-mer for the $\mathrm{C}$ allele. Even the T-specific and G-specific cleavages yield discernible changes, since the $\mathrm{C}$-allele is $24 \mathrm{Da}$ less massive than the A-allele, a peak shift that is easily detected in the low mass portion of the mass spectrum. Any one of these reactions would be sufficient to detect this polymorphism, but taken together the precise location can be determined since in most instances there is only one way to reconcile all four peak patterns.

The single-stranded nucleic acid is produced by transcription-a very reliable, economical, and process-friendly method. A T7 RNA polymerase promoter can be attached to either end of an amplicon during DNA amplification using the threeprimer system depicted in Figure 4. Target-specific amplification primers are used, each with a slightly different sequence tag at

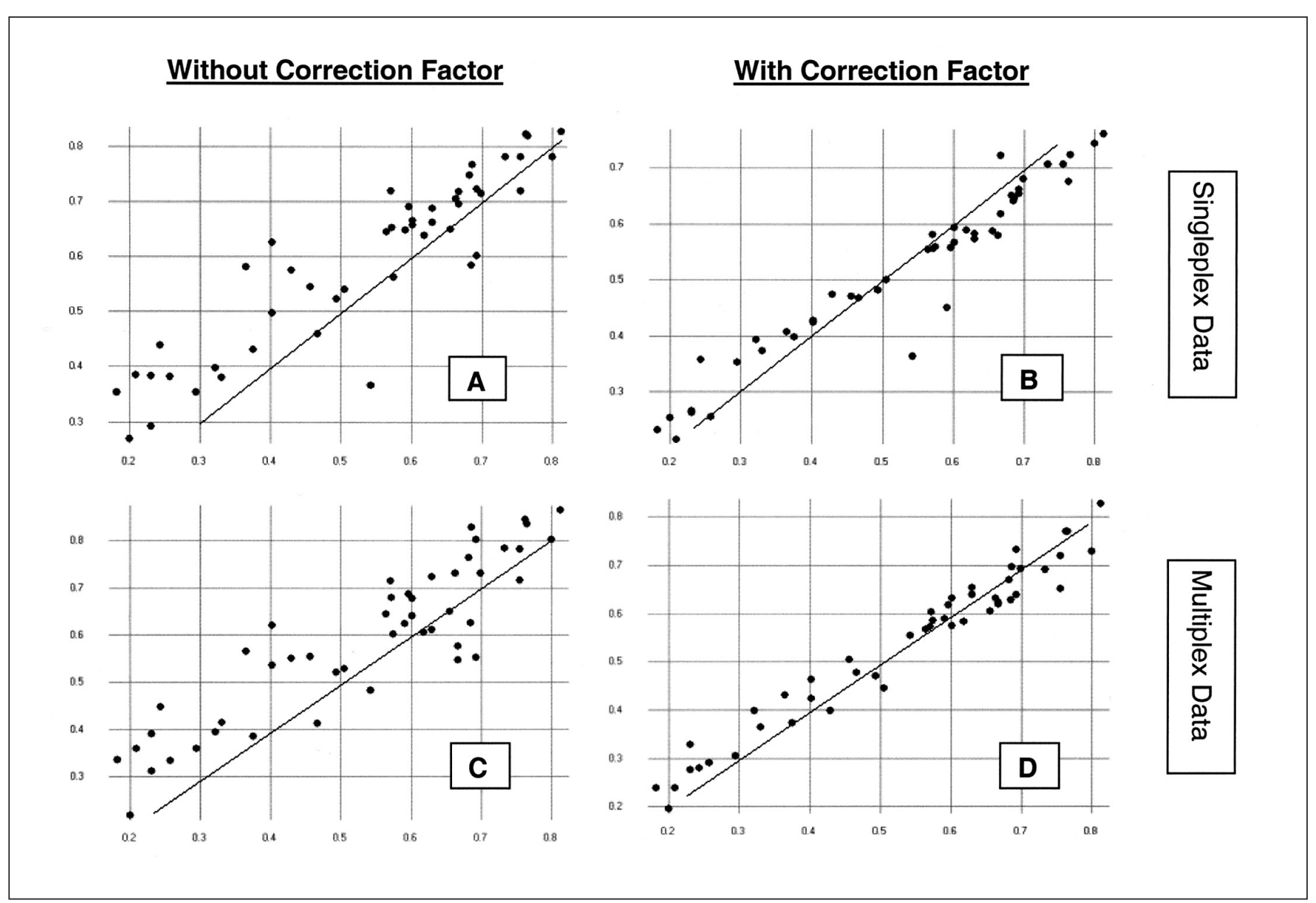

Figure 2. Allelic frequency (AF) determination: singleplex hME versus multiplex hME, with and without correction factor. 48 different hME assays were genotyped on 93 DNA samples as 4-plex reactions. The resulting allelic frequency was compared to allelic frequency results obtained from pooled DNA experiments, either performed as singleplex or multiplex (=4-plex) hME. (A) AF obtained from singleplex hME on pooled DNA. (B) AF obtained from singleplex hME on pooled DNA, correction factor was used for AF determination. (C) AF obtained from multiplex hME on pooled DNA. (D) AF obtained from multiplex hME on pooled DNA, correction factor was used for AF determination. X-Axes, allelic frequency obtained from genotyping. Y-Axes, allelic frequency obtained from pooled DNA experiments. 


\begin{tabular}{|c|c|c|}
\hline \multicolumn{3}{|c|}{ 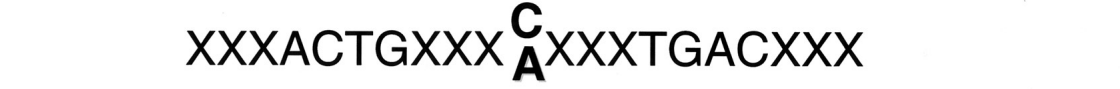 } \\
\hline \multirow{2}{*}{$\begin{array}{c}\text { Base- } \\
\text { specific } \\
\text { cleavage } \\
\text { at: } \\
\end{array}$} & \multicolumn{2}{|c|}{ Resulting analytic fragment(s) of } \\
\hline & A-allele: & C-allele: \\
\hline A & CTGXXXA XXXTGA & \begin{tabular}{|l|} 
CTGXXXCXXXTGA \\
\end{tabular} \\
\hline C & TGXXXAXXXTGAC & \begin{tabular}{|l|l|} 
TGXXXC XXXTGAC \\
\end{tabular} \\
\hline$T$ & $\frac{\text { GXXXAXXXT }_{A} \text { is } 24 \mathrm{Da}>\mathrm{C}}{}$ & GXXXCXXXT \\
\hline $\mathbf{G}$ & $\frac{\underset{A}{\text { XXXAXXXT } 24 \mathrm{Da}}>\mathrm{C}}{\mathrm{C}}$ & XXXCXXXTG \\
\hline
\end{tabular}

Figure 3. Base-specific fragmentation yields a distinctly different pattern for different alleles. The sequence shown contains an $\mathrm{A} / \mathrm{C}$ polymorphism as indicated in boldface type. For each base-specific fragmentation there is a distinct change in the fragment(s) produced. The A-specific fragmentation yields two fragments for the Aallele (a 7-mer and a 6-mer); the C-allele yields only one fragment, a 13-mer. The converse is true of the C-specific fragmentation, with a 13-mer produced for the Aallele and for the C-allele, a 6-mer and a 7-mer. For both the T-specific and G-specific fragmentations, the length of the fragments produced for each allele is the same, but the composition and therefore the mass of fragments differ. In each case, the A-allele containing fragment is $24 \mathrm{Da}$ more massive than the C-allele containing fragment, which causes an easily resolvable shift in the mass spectrum. For the example given there are five mass peaks that will differ between the two alleles.

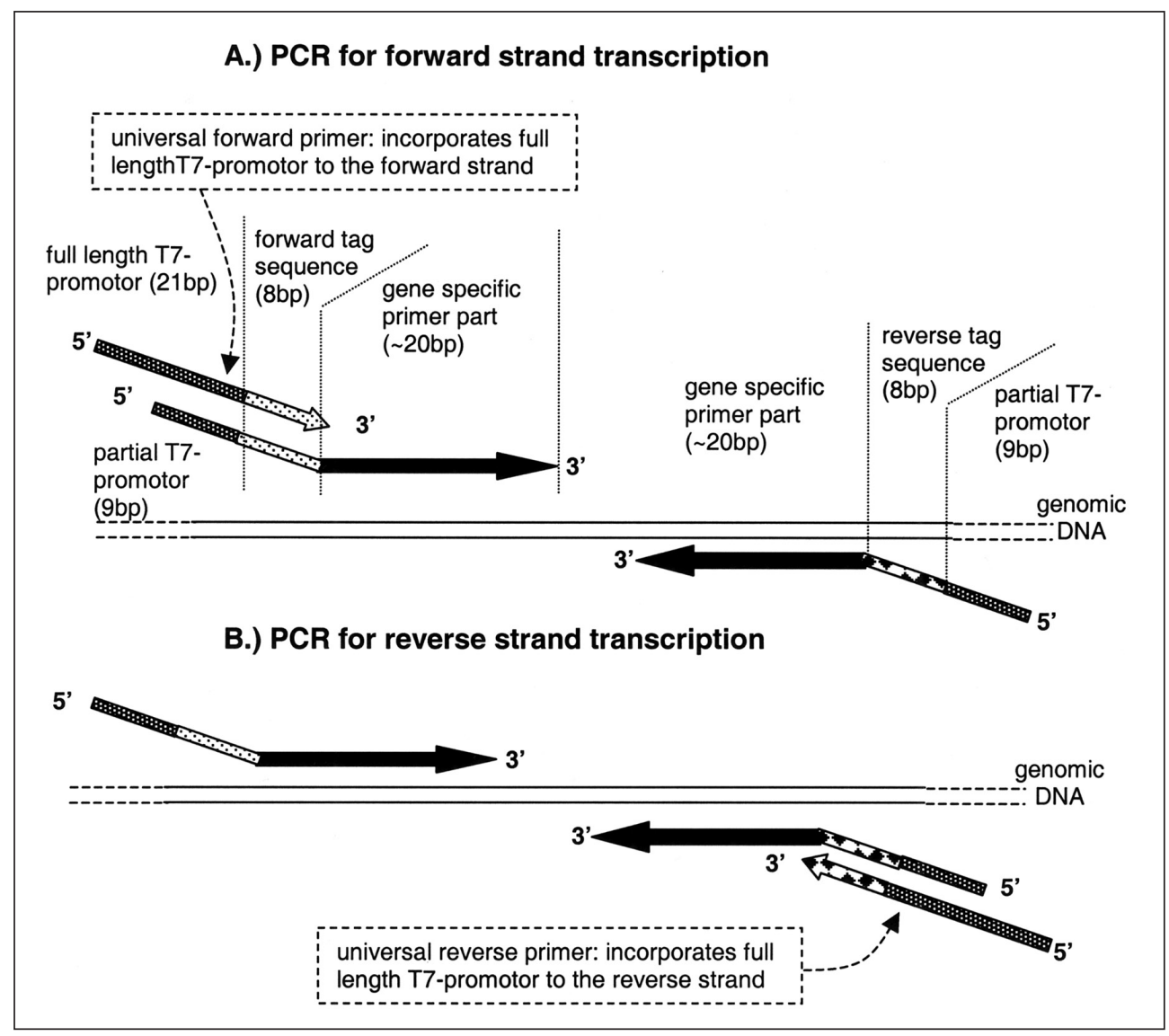

Figure 4. Three primer system used during PCR for fragmentation reactions. As a transcription reaction follows the polymerase chain reaction (PCR), the T7RNA polymerase promotor sequence has to be incorporated during the PCR step. This can be done by either attaching the T7-promotor directly to the 5 ' end of the sequence specific primer (either forward or reverse primer-not shown in this figure), or by using a three primer system as depicted. The advantage of a three primer system is cost reduction, as the universal forward and reverse primer can be used for any given assay and only two sequence specific primers are needed per assay. Each sequence specific primer has an additional 17 bases attached to the $5^{\prime}$ end. Those 17 bases serve as annealing sites for the universal primers. Of those 17 bases, 9 bases are part of the T7-promoter and 8 bases contain the "tag sequence," which distinguishes the binding site for the universal forward or reverse primer. 
the $5^{\prime}$ end. By including a universal forward T7 primer in the reaction, amplicons are created that produce + transcripts; by substituting a universal reverse T7 primer into the reaction, amplicons are created that produce - transcripts. In high-throughput mode, it is recommended to simply run two + strand reactions and two - strand reactions rather than distribute transcripts after they are produced. The two + strands are fragmented using an $\mathrm{RNase}$ reaction specific for $\mathrm{C}$ residues in one well and a second reaction specific for $U$ residues in the other well. G-specific and A-specific cleavages are deduced by simply running the Cspecific and U-specific reactions, respectively, on the - strands.

One of the great advantages of the fragmentation approach for discovery of genetic variation is the clarity of the signal produced. This permits targeted discovery using amplicons (rather than clones) and fully automated interpretation of the results. An example of this is shown in Figure 5. A 500-bp amplicon from intron 10 of the CETP gene was produced from each of 12 individuals, transcribed, and subjected to T-specific fragmenta-

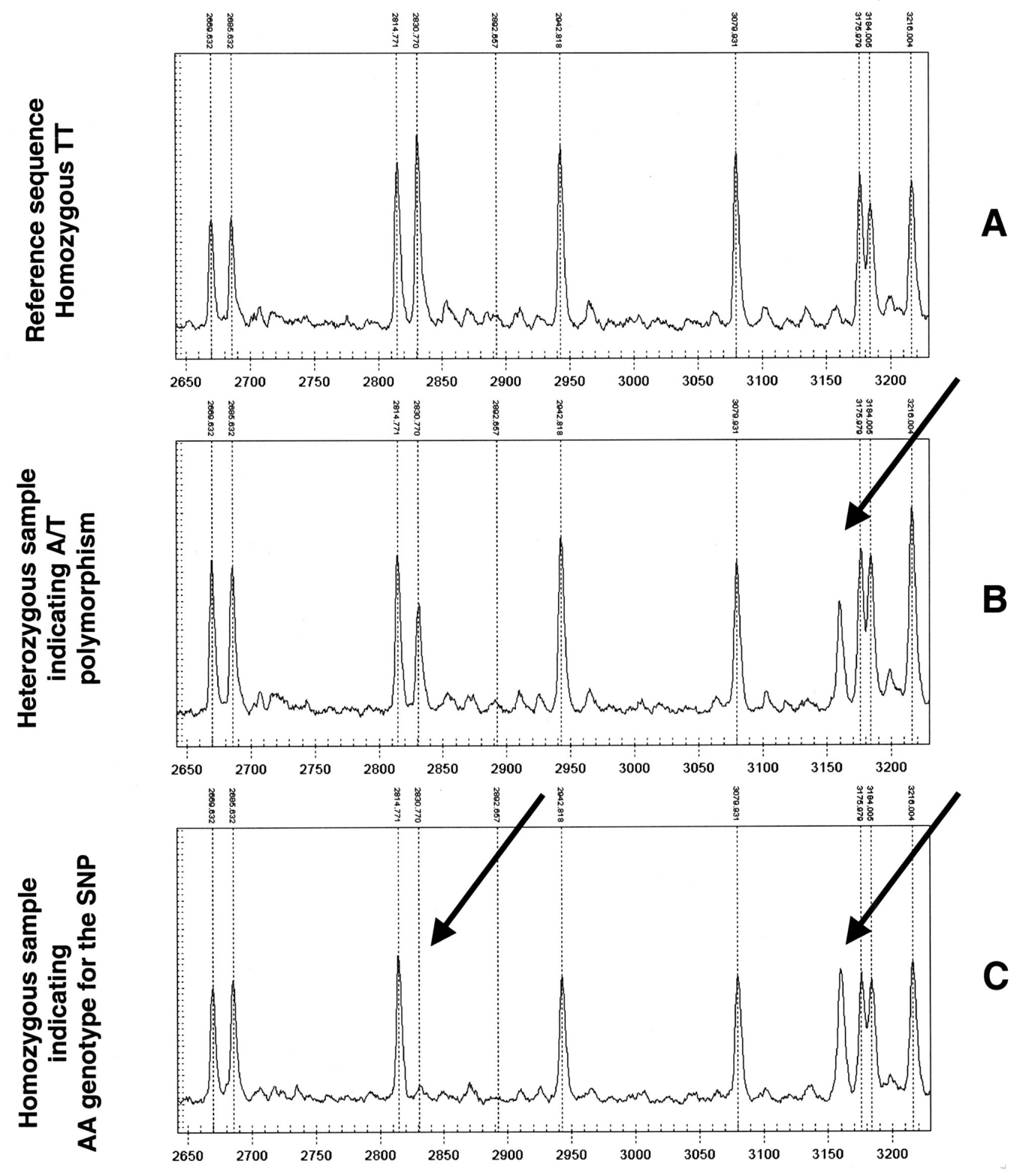

Figure 5. Base-specific cleavage of intron 10 of the CETP gene reveals a new SNP. Panel A displays a mass spectrum from a T-specific fragmentation (or U-specific, since the actual fragmented nucleic acid was RNA) of in vitro transcripts of an intron of the CETP gene. The pattern displayed is consistent with the Ensembl reference sequence. Panel B shows the same reaction run on a different individual. The arrow indicates a new peak at $3159 \mathrm{Da}$, not predicted by the reference sequence. Note also that the mass peak at $2830.7 \mathrm{Da}$ is diminished in intensity compared to the spectrum in panel A. Panel C is a spectrum from yet another individual. The peak at $3159 \mathrm{Da}$ is greater in intensity than the same peak in panel $\mathrm{B}$; also, the peak at $2830.7 \mathrm{Da}$ is absent. Because of the precise mass determinations, it is possible to assign an $\mathrm{A} / \mathrm{T}$ genotype to the individual represented in panel $\mathrm{B}$, and an $\mathrm{A} / \mathrm{A}$ genotype to the individual in panel $\mathrm{C}$. 
tion. The partial spectrum displayed in panel A corresponds precisely to the predicted peak pattern based on the Ensembl sequence; all expected peaks (marked by dotted lines) are present and no unexpected peaks are seen. (The dotted line at 2882.6 Da marks where a rare artifact caused by abortive cycling during transcription can occur; it is not present in this reaction.) Two of the twelve individuals showed different patterns. The spectrum in panel B has an unexpected peak at $3159 \mathrm{Da}$; furthermore, the peak at 2830.7 Da has a significantly reduced signal intensity. Since no predicted peaks are absent, this is consistent with one of the homologues of this individual having a nucleotide substitution at a $\mathrm{T}$ residue, thereby rendering it resistant to cleavage and resulting in the new signal at the higher mass. Panel $\mathrm{C}$ has the same unexpected peak at $3159 \mathrm{Da}$, but its relative intensity is greater, and the peak at $2830.7 \mathrm{Da}$ is completely absent; this individual is homozygous for the heretofore unknown SNP. Because of the accuracy of the MassARRAY System and existence of a reference sequence, this polymorphism can be automatically called as an $\mathrm{A} / \mathrm{T}$ transversion. The design of an $\mathrm{hME}$ assay for subsequent high-throughput genotyping is simple, since both the location and nature of the newly discovered SNP is known.

\section{MULTIPLEXED hME GENTOTYPING}

The final step in the strategy described here is to take all of the SNPs of interest (i.e., the original SNPs that showed a statistically significant difference in allelic frequency between pools; known SNPs identified in nearby regions using the RealSNP.com Web site; and SNPs discovered in those nearby regions using base-specific fragmentation) and combine them into multiplexed assays (10). These assays are then taken across all of the individuals constituting the two phenotypically defined pools.

Although uniplex hME assays are designed such that the mass of each product differs from the other products by at least the mass of a single nucleotide (approximately $300 \mathrm{Da}$ ), much smaller mass differences can be used without compromising genotyping accuracy. This allows for the routine design of multiplexed SNP assays using SpectroDESIGNER software, the same software used for uniplex hME designs. All hME genotyping reactions are single-tube, addition-only reactions, therefore primers for multiplexed amplification and accompanying multiplexed hME reactions are designed together to ensure compatibility. Plate configuration, optimal terminator mixes, and

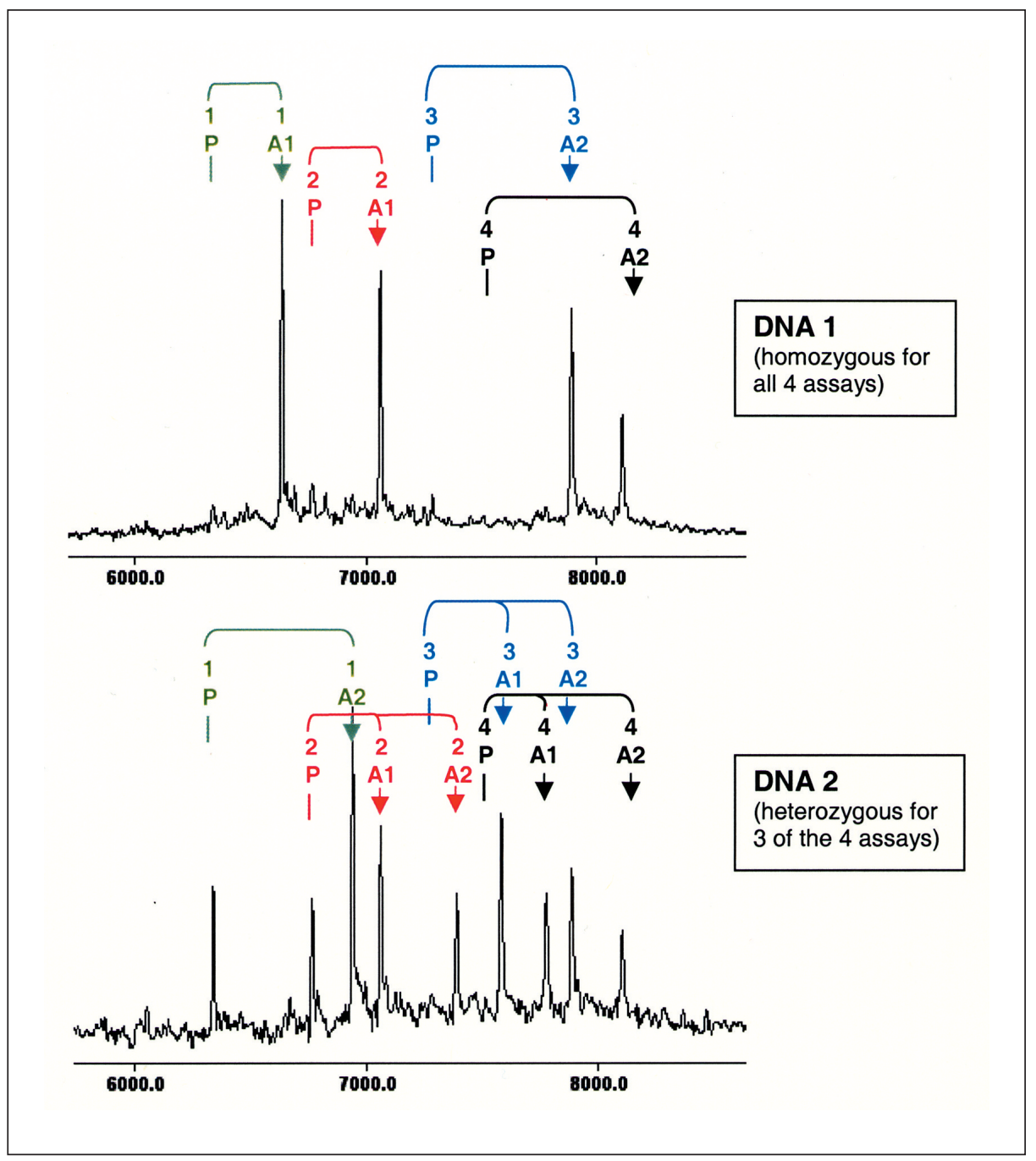

Figure 6. Two MALDI-TOF MS spectra examples of one and the same hME 4-plex reaction. The first spectra (DNA 1) shows a DNA sample that is homozygous for all 4 SNPs whereas the second spectra (DNA 2) shows a DNA sample that is heterozygous for 3 of the 4 SNPs. $1 \mathrm{P}$ to $4 \mathrm{P}$, unextended hME primer from assay 1 to $4.1 \mathrm{~A} 1$ to $4 \mathrm{~A} 1$, Allele 1 for assays 1 to 4 . $1 \mathrm{~A} 2$ to $4 \mathrm{~A} 2$, Allele 2 for assays 1 to 4 . 
purchase requisitions are also generated through SpectroDESIGNER. All multiplexed reactions are performed under uniform buffer and cycling conditions and usually consist of four to six SNP assays per multiplex.

An example of a 4-plex reaction run against DNA from two individuals is shown in Figure 6. For DNA 1, the individual is homozygous at all four SNP loci; for DNA 2, the individual is homozygous at one locus (SNP 1) and heterozygous at three loci (SNPs 2, 3, and 4).

In both cases one can see how products and primers from different assays can interleave with each other to accommodate the multiplexing, yet yield easily resolved mass signals. As with all SEQUENOM applications, the scoring and reporting of the assay results are completely automated.

\section{SUMMARY AND DISCUSSION}

The strategy presented here is a simple and straightforward one that is possible because of the speed, accuracy, and affordability of the hME reaction on the MassARRAY System. The beginning step of using pooled DNA samples to determine and compare allelic frequencies is critical. By way of example, consider running assays for 10000 SNPs over two phenotypically defined populations, each containing 300 individuals; such a study would require 6000000 individual genotyping reactions. This number of reactions could be reduced to 56000 reactions by following the strategy outlined in this paper. First, run 20000 reactions (10000 SNPs across two pools) to determine allelic frequency differences between the phenotypically defined pools. Next, use only those SNPs that showed significant differences in allelic frequency (e.g., $3 \%$ or 300 SNPs) for individual genotyping (36000 reactions: 300 SNPs in sixty 5-plexes across two populations of 300 individuals each). The total number of reactions needed is 20000 for the allelic frequency determinations plus 36000 for genotyping of individuals, or a total of 56000 reactions. Instrument time needed to do such a study would be just a few days versus over three months for genotyping each individual (three weeks if those reactions were run as 5plexes). Although there may be times when the expense and effort of genotyping large numbers of individuals is worthwhile (e.g., in creating a searchable database of both genotypes and phenotypes for a disease franchise), in many cases the strategy presented here provides a viable and affordable method of discovering disease markers - and perhaps causative SNPs.

Of special note is the base-specific fragmentation method of SNP discovery developed by SEQUENOM and Methexis Genomics. This method provides a practical, fully automated method for high-throughput discovery of genetic variation whenever a reference sequence is known. One of the great advantages of the base-specific fragmentation approach for the discovery of genetic variation is the clarity of the signal produced. This permits very targeted discovery using amplicons (rather than clones) and fully automated interpretation. This provides a distinct advantage over dideoxy sequencing. In conventional sequencing, sequences from an amplicon produced from a heterozygote would yield two signals at the same position on the gel, which would likely require manual inspection by a skilled user. In base-specific fragmentation, the signals are dramatically shifted and unmistakable, allowing for fully automated, highthroughput discovery of SNPs.

The combination of hME determination of allelic frequencies, SNP discovery by base-specific fragmentation, and genotyping individuals using multiplexed hME, create a powerful suite of applications for the discovery and analysis of genetic variation, all of which can be done on the MassARRAY System.

\section{REFERENCES}

1.Buetow, K.H., M. Edmonson, R. MacDonald, R. Clifford, P. Yip, J. Kelley, D.P. Little, R. Strausberg, et al. 2001. High-throughput development and characterization of a genome-wide collection of gene-based single nucleotide polymorphism markers by chip-based matrix-assisted laser desorption/ionization time-of-flight mass spectrometry. Proc. Natl. Acad. Sci. USA 98:581584

2.Collins, A., C. Lonjou, and N.E. Morton. 1999. Genetic epidemiology of single-nucleotide polymorphisms. Proc. Natl Acad. Sci. USA 96:1517315177.

3.Karas, M. and F. Hillenkamp. 1988. Laser desorption ionization of proteins with molecular weights exceeding 10000 Daltons. Anal. Chem. 60:22992301 .

4.Kruglyak, L. 1999. Prospects for whole genome linkage disequilibrium mapping of common disease genes. Nat. Genet. 22:139-144.

5.Little, D.P., A. Braun, M.J. O’Donnell, and H. Koster. 1997. Mass spectrometry from miniaturized arrays for full comparative DNA analysis. Nat. Med. 3:1413-1416.

6.Riley, J.H., C.J. Allan, E. Lai, and A. Roses. 2000. The use of single nucleotide polymorphisms in the isolation of common disease genes. Pharmacogenomics 1:39-47.

7.Risch, N. and K. Merinkangas. 1996. The future of genetic studies of complex human diseases. Science 273:1516-1517.

8.Sanger, F., S. Nicklen, and A.R. Coulson. 1977. DNA sequencing with chain terminating inhibitors. Proc. Natl. Acad. Sci. USA 12:5463-5467.

9.Shchepinov, M.S., M.F. Denissenko, K.J. Smylie, R.J. Worl, A.L. Leppin, C.R. Cantor, and C.P. Rodi. 2001. Matrix-induced fragmentation of P3'N5' phosphoramidate-containing DNA: high-throughput MALDI-TOF analysis of genomic sequence polymorphisms. Nucleic Acids Res. 29:386472 .

10.Storm, N., B. Darnhofer-Demar, D. van den Boom, and C.P. Rodi. MALDITOF mass spectrometry-based SNP genotyping. Methods Mol. Biol. (In press).

11.von Wintzingerode, F., S. Böcker, C. Schlötelburg, N.H.L. Chiu, N. Storm, C. Jurinke, C.R. Cantor, U.B. Göbel, and D. van den Boom. Basespecific fragmentation of amplified 16S rRNA genes analyzed by mass spectrometry: a novel tool for rapid bacterial identification. Proc. Natl. Acad. Sci. USA (In press).

\section{Address correspondence to:}

Dr. Dirk van den Boom

SEQUENOM

3595 John Hopkins Court

San Diego, CA 92121, USA

e-mail:dvandenboom@sequenom.com 